\title{
Estudo das Pressões no Interior dos Balonetes de Tubos Traqueais *
}

\section{Study of Tracheal Tube Intra-Cuff Pressure}

André Galante Alencar Aranha ${ }^{1}$; Vicente Forte ${ }^{2}$; João Aléssio Juliano Perfeito ${ }^{3}$; Luiz Eduardo Villaça Leão ${ }^{4}$; Carlos Jogi Imaeda ${ }^{5}$; Yara Juliano ${ }^{6}$

\section{RESUMO}

Aranha AGA, Forte V, Perfeito JAJ, Leão LEV, Imaeda CJ, Juliano $Y$ - Estudo das Pressões no Interior dos Balonetes de Tubos Traqueais

JUSTIFICATIVA E OBJETIVOS: Como não é rotineiro o controle da pressão no interior dos balonetes de tubos traqueais, e não há descrição detalhada na literatura de como mantê-la abaixo dos $30 \mathrm{cmH}_{2} \mathrm{O}$ sem utilização de manômetro, decidiu-se verificar as pressões no interior de balonetes de tubos traqueais em pacientes sob intubação traqueal na unidade de terapia intensiva e no cento cirúrgico, testando manobra para manter a pressão no balonete abaixo de 30 $\mathrm{CmH}_{2} \mathrm{O}$, mas em níveis mínimos necessários para ciclagem do ventilador sem perda do volume corrente.

MÉTODO: Foram estudadas as pressões no interior de balonetes de tubos traqueais de 50 pacientes sob intubação traqueal na unidade de terapia intensiva (Grupo I) e 72 pacientes sob intubação traqueal no centro cirúrgico (Grupo II). Testou-se uma manobra para obter a pressão mínima no interior do balonete do tubo traqueal, necessária para adequada ventilação, sem vazamento de ar. Registrou-se a pressão inicial (P1) no interior dos balonetes dos tubos traqueais utilizando-se manômetro digital graduado em centímetros de água, acoplado a seringa de $15 \mathrm{ml}$. Aspirou-se secreção da orofaringe. Com o meato acústico externo do examinador próximo da boca do paciente entre 10 e $20 \mathrm{~cm}$, conectou-se o manômetro ao balonete, que foi esvaziado lentamente, até se ouvir ruído em sopro, pelo vazamento do volume corrente no período inspiratório da ventilação artificial. Neste momento, encheu-se lentamente o balonete até o desaparecimento do ar que restou na seringa do manômetro (V).

\footnotetext{
* Recebido do (Received from) dos Hospitais da Real e Benemérita Sociedade Portuguesa de Beneficência e Professor Edmundo Vasconcelos e apresentado como Tese de Mestrado na Universidade Federal de São Paulo, Escola Paulista de Medicina (UNIFESP), São Paulo, SP

1. Pós-Graduando da Disciplina de Cirurgia da UNIFESP; Titular da Sociedade Brasileira de Cirurgia Torácica

2. Docente e Chefe da Disciplina de Cirurgia Torácica da UNIFESP; Titular da Sociedade Brasileira de Cirurgia Torácica

3. Professor Assistente da Disciplina de Cirurgia Torácica da UNIFESP, Titular da Sociedade Brasileira de Cirurgia Torácica

4. Docente Coordenador do Curso de Pós-Graduação em Medicina da UNIFESP; Titular da Sociedade Brasileira de Cirurgia Torácica

5. Assistente da Disciplina de Cirurgia Torácica da UNIFESP; Titular da Sociedade Brasileira de Cirurgia Torácica

6. Docente da Disciplina de Bioestatística da UNIFESP
}

Apresentado (Submitted) em 25 de fevereiro de 2002

Aceito (Accepted) para publicação em 08 de abril de 2003

Endereço para correspondência (Correspondence to)

Dr. André Galante Alencar Aranha

Av. Siqueira Campos 634/113 Boqueirão

11045-200 Santos, SP

(c) Sociedade Brasileira de Anestesiologia, 2003 ruído. Anotou-se a pressão final $(P 2)$ do balonete e o volume de

RESULTADOS: As médias das pressões $P 1$ nos grupos / e II foram 85,3 e 56,2 $\mathrm{cmH}_{2} \mathrm{O}$, respectivamente. As médias de pressões $P 2$ nos grupos / e ll foram 26,7 e 15,5 $\mathrm{cmH}_{2} \mathrm{O}$ respectivamente. Após a manobra testada, o desvio padrão baixou de 56,3 para 8,2 no grupo I, e de 48 para 6,7 no grupo II. No grupo I, a manobra reduziu o volume e a pressão dos balonetes em $100 \%$ dos pacientes e no grupo II, em $97,3 \%$.

CONCLUSÕES: Os dois grupos apresentaram pressões no interior dos balonetes em níveis acima do necessário para ciclagem do ventilador sem perda do volume corrente. A manobra para manter a pressão no interior do balonete em níveis inferiores a $30 \mathrm{cmH}_{2} \mathrm{O}$ foi simples e de pequeno custo.

Unitermos: EQUIPAMENTOS: tubo traqueal; INTUBAÇÃO TRAQUEAL; TÉCNICAS DE MEDIÇÃO: pressão do balonete

\section{SUMMARY}

Aranha AGA, Forte V, Perfeito JAJ, Leão LEV, Imaeda CJ, Juliano $Y$ - Study of Tracheal Tube Intra-Cuff Pressure

BACKGROUND AND OBJECTIVES: Since controlling tracheal tube cuffs internal pressure is unusual and there is no detailed description in the literature on how to maintain it below 30 $\mathrm{cmH}_{2} \mathrm{O}$ without manometer, this study aimed at checking tracheal tube intra-cuff pressures in intensive care unit and operating room patients. A maneuver was tested to keep intra-cuff pressure below $30 \mathrm{cmH}_{2} \mathrm{O}$, but at minimum levels needed for ventilator cycling with no tidal volume leakage.

METHODS: Tracheal tube intra-cuff pressures were evaluated in 50 intensive care unit intubated patients (Group I) and 72 intubated patients in the operating room (Group II). A maneuver was tested to obtain the minimum tracheal tube intra-cuff pressure to maintain adequate ventilation with no air leakage. Initial tracheal tube intra-cuff pressure $(P 1)$ was recorded using a gaged digital manometer $\left(\mathrm{cmH}_{2} \mathrm{O}\right)$ coupled to a $15-\mathrm{ml}$ syringe. Oropharynx secretion was aspirated. With the investigator's external acoustic meatus positioned $10-20 \mathrm{~cm}$ apart from patient's mouth and cuff connected to the manometer, cuff was slowly deflated until a murmur sound was heard, determined by tidal volume leakage during the inspiratory period of artificial ventilation. At this moment, cuff was slowly inflated until murmur disappearance. Final intra-cuff pressure $(P 2)$ and the remaining air volume in the manometer syringe (V) were recorded.

RESULTS: Mean P1 values in groups I and I/ were 85.3 and $56.2 \mathrm{CmH}_{2} \mathrm{O}$, respectively. Mean P2 values in groups I and II were 26.7 and $15.5 \mathrm{cmH}_{2} \mathrm{O}$, respectively. After the maneuver, standard deviation decreased from 56.3 to 8.2 in group I, and from 48 to 6.7 in group II. Maneuver has decreased cuff volume and pressure in $100 \%$ of group I patients, and in $97.3 \%$ of group II patients.

CONCLUSIONS: Both groups had intra-cuff pressures higher than necessary to keep ventilator cycling with no tidal volume leakage. Maneuver to keep intra-cuff pressure below $30 \mathrm{cmH}_{2} \mathrm{O}$ was simple and cheap.

Key Words: EQUIPMENTS: tracheal tube; MEASUREMENT TECHNIQUES: cuff pressure; TRACHEAL INTUBATION 


\section{INTRODUÇÃO}

A s estenoses traqueais podem ter inúmeras etiopatogenias, entretanto, a mais freqüente está relacionada com a intubação traqueal ${ }^{1-8}$.

A incidência de estenoses laríngea e traqueal após intubação varia de $1,5 \%$ até $19 \%{ }^{9-11}$

Cooper e Grillo experimentaram em cães, e posteriormente testaram no homem ${ }^{1}$, tubo traqueal com balonete de látex, facilmente distensível, mais maleável, que se adaptava ao formato da traquéia, necessitando menor pressão para evitar escape do fluxo de ar. Após o desenvolvimento destes tubos com balão de baixa pressão, no início dos anos 70 , como auxílio da bioengenharia, houve sensível diminuição das estenoses traqueais pós intubação traqueal ${ }^{1,2,7,11}$. Entretanto, mesmo balonetes de baixa pressão, quando insuflados com volume excessivo, têm as pressões no seu interior elevadas em níveis que podem ser danosos para a traquéia ${ }^{12,13}$.

Nordin, em 1977, demonstrou em coelhos que a pressão no balonete de tubo traqueal acima de $30 \mathrm{mmHg}\left(40 \mathrm{cmH}_{2} \mathrm{O}\right)$ causa isquemia da mucosa e, por isso, recomendou que essa pressão ficasse abaixo de $20 \mathrm{mmHg}\left(26 \mathrm{cmH}_{2} \mathrm{O}\right)^{4}$.

Pippin e col., em 1983, enviaram um questionário a 152 unidades de terapia intensiva da Inglaterra indagando se a medida das pressões no interior dos balões dos tubos traqueais era feita rotineiramente. Observaram que em $83,2 \%$ dos questionários a resposta foi negativa ${ }^{14}$.

Estenose traqueal após intubação pode ocorrer em paciente sob intubação traqueal com tubo com balonetes de baixa pressão, mesmo que por períodos curtos, até de poucas horas $^{7,15,16}$.

Forte, em 1996, apresentou casuística de 250 pacientes submetidos a traqueoplastias, no período de março de 1969 a março de 1996, para correção de estenose traqueal após intubacão, e atribuiu a incidência de $80 \%$ das lesões ao balonete do tubo traqueal ${ }^{7}$.

O objetivo deste estudo foi verificar as pressões no interior dos balonetes de tubos traqueais em pacientes internados em unidade de terapia intensiva e nos submetidos à cirurgia, e testar uma manobra simples, rápida e de baixo custo, para adequar a pressão no balonete em nível mínimo, e sempre que possível abaixo de $30 \mathrm{cmH}_{2} \mathrm{O}$.

\section{MÉTODO}

Após aprovação Institucional do Hospital São Joaquim da Real e Benemérita Sociedade Portuguesa de Beneficência, no período de julho a dezembro de 1997, escolheu-se, aleatoriamente, 50 pacientes nas Unidades de Terapia Intensiva (Grupo I). Após aprovação da Comissão de Ética do Hospital Professor Edmundo Vasconcelos, foi estudado no centro cirúrgico deste Hospital, um segundo grupo de 72 pacientes no período de maio a agosto de 1999 (Grupo II).

No grupo I, em dias aleatórios, verificou-se a pressão dos balonetes. Os tubos de intubação traqueal eram da marca Rush $^{\circledR}$, com diâmetro interno de 7 a $9 \mathrm{~mm}$, sendo todos com balonete de baixa pressão. No grupo II, estudaram-se 72 pa- cientes intubados para procedimentos cirúrgicos sob anestesia geral. Verificou-se também a pressão dos balonetes. Foram sorteadas três salas das setes do centro cirúrgico, no período da manhã e três no da tarde. Foram excluídos da avaliação pacientes sob anestesia local e anestesiados com óxido nitroso, pois este passa pelo material do balonete alterando sua pressão durante a anestesia. Todos foram intubados com tubos da marca Rush ${ }^{\circledR}$, com balonete de baixa pressão.

Nos dois grupos, os pacientes foram intubados por diferentes profissionais. A injeção de ar no balonete do tubo no momento da intubação traqueal e a manutenção do grau de seu enchimento foram realizadas conforme critérios pessoais de cada profissional responsável, que não tinha conhecimento sobre a realização do estudo.

Técnica de Mensuração da Pressão no Interior do Balonete dos Tubos Traqueais

Nos dois grupos, mediu-se as pressões no interior dos balonetes dos tubos de intubação traqueal com manômetro (P-Gauge da Mallinckrodt Medical ${ }^{\circledR}$ ). Este aparelho tem uma seringa graduada em $15 \mathrm{~cm}^{3}$, acoplada a um manômetro digital graduado em $\mathrm{cmH}_{2} \mathrm{O}$.

Em cada paciente a medida de pressão no interior do balão foi realizada da seguinte maneira:

a) Aspiração da secreção orofaríngea do paciente;

b) Conexão do aparelho ao balonete externo do tubo;

c) Verificação da pressão no interior do balonete dos tubos traqueais, a qual definiu-se como pressão inicial (P1);

d) Esvaziou-se lentamente o balonete e, ao mesmo tempo, o examinador manteve seu meato acústico externo a uma distância entre 10 e $20 \mathrm{~cm}$ da boca do paciente. Desta forma o examinador podia ouvir um ruído em sopro quando parte do volume corrente vazava ao redor do balonete no período inspiratório da ventilação artificial;

e) Em seguida, encheu-se lentamente o balonete até que o ruído não fosse mais audível;

f) Anotou-se a pressão no interior do balonete que foi necessária para cessar o vazamento. Esta pressão foi considerada a pressão final (P2);

g)Após a parada do vazamento de ar, mediu-se o volume de ar que ficou na seringa, o qual definiu-se como o volume desnecessário $(\mathrm{V})$.

Nas primeiras dez manobras realizadas, verificou-se o tempo gasto para completar todas as etapas.

Para a análise dos resultados, na comparação das pressões inicial e final no interior dos balonetes, utilizou-se o teste não paramétrico de Wilcoxon. Foram colocados, além da mediana, $\mathrm{P}_{25}$ e $\mathrm{P}_{75}$, os valores da média e desvio padrão para se ter idéia da variabilidade dos dados.

Fixou-se o nível de rejeição da hipótese de nulidade em 0,05 ou $5 \%(p<0,05)$, assinalando-se com asterisco os valores significantes. 


\section{RESULTADOS}

O grupo I foi composto por 18 mulheres (36\%), 32 homens (64\%), com idades entre 22 e 99 anos, sendo a média das idades de 62,1 anos. As intubações foram devidas a pós-operatório em cirurgia cardiovascular em 27 casos, broncopneumonias em cinco casos, pós-operatórios de cirurgia geral em cinco casos, infarto do miocárdio em três casos, e outras causas em 10 casos. O tempo de intubação variou de algumas horas a 23 dias.

O grupo Il foi composto por 42 homens $(58,3 \%)$ e 30 mulheres $(41,7 \%)$, com idades entre 15 e 68 anos, com média de idade de 45,2 anos. O tempo de intubação variou de $1 \mathrm{~h} 40$ minutos a 6 h20 minutos.

O tempo para realização de todas as etapas descritas para verificar a pressão no interior do balonete foi menor do que três minutos. Os valores das pressões iniciais foram sempre maiores do que as pressões finais após a realização da manobra já descrita. Na figura 1, estão ilustrados os valores de pressão inicial e após a manobra nos pacientes do grupo I. $\mathrm{Na}$ figura 2 encontram-se as pressões iniciais e finais verificadas nos pacientes do grupo II. As figuras mostram a grande variabilidade das pressões iniciais, tendo como conseqüência um grande desvio padrão.

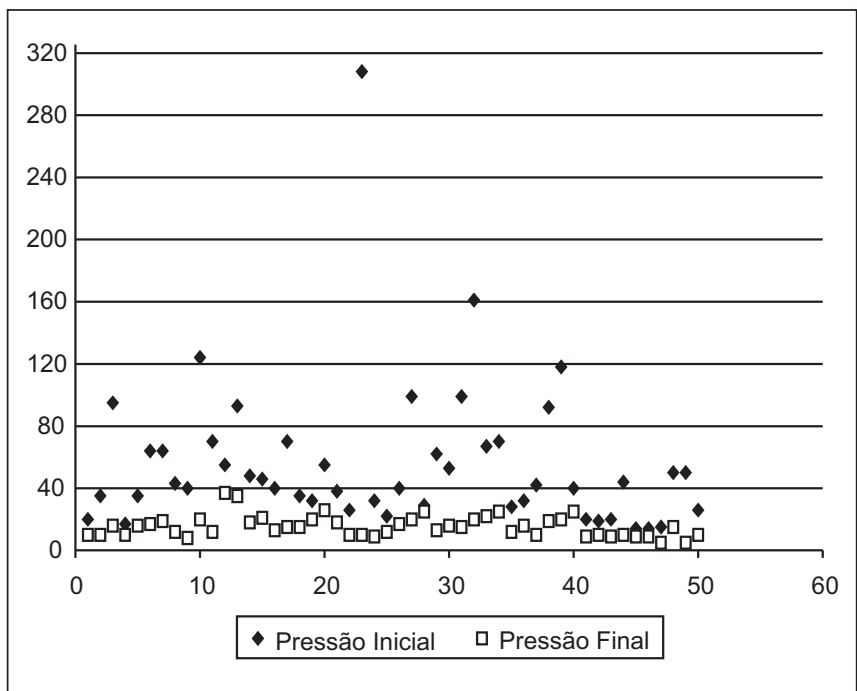

Figura 1 - Pressões Inicial e Final, em $\mathrm{cmH}_{2} \mathrm{O}$, no Interior dos Balonetes dos Tubos Traqueais dos Pacientes do Grupo I

No grupo I apenas 12 (24\%) balonetes apresentaram pressões iniciais em níveis menores do que $30 \mathrm{cmH}_{2} \mathrm{O}$. Nos 50 pacientes (100\%), reduziram-se a pressão e o volume de ar no interior dos balonetes, sendo que apenas em dois pacientes as pressões não ficaram com pressões abaixo de $30 \mathrm{cmH}_{2} \mathrm{O}$ após a manobra. No grupo II, cinco $(6,9 \%)$ balonetes estavam com pressão menor do que $30 \mathrm{cmH}_{2} \mathrm{O}$. Dos 72 pacientes, em apenas dois $(2,7 \%)$ não houve retirada de ar do balonete, e em $23(31,9 \%)$ pacientes não se conseguiu manter pressões inferiores a $30 \mathrm{cmH}_{2} \mathrm{O}$, sem que houvesse escape de ar durante a ventilação.

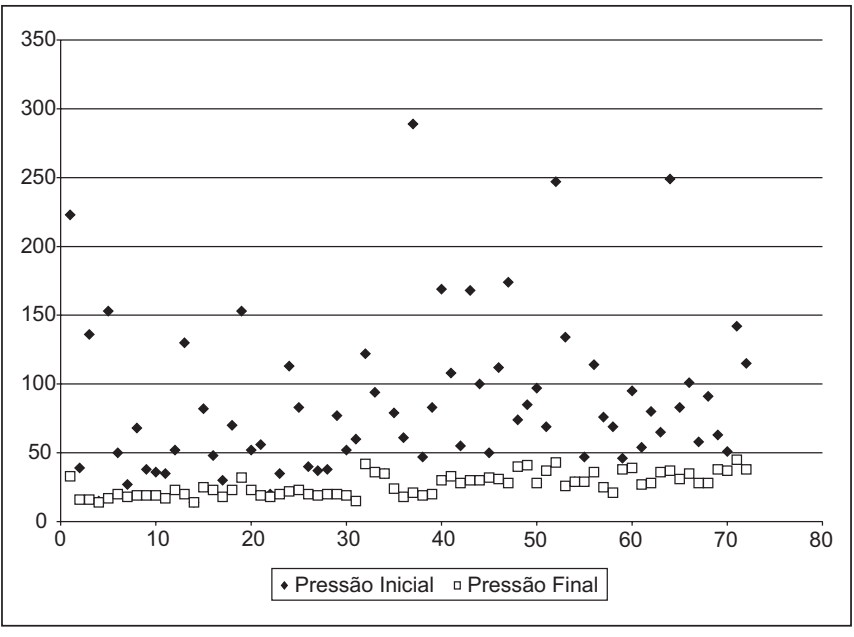

Figura 2 - Pressões Inicial e Final, em $\mathrm{cmH}_{2} \mathrm{O}$, no Interior dos Balonetes dos Tubos Traqueais dos Pacientes do Grupo II

A média, o desvio padrão, a mediana, a $\mathrm{P}_{25}$ e a $\mathrm{P}_{75}$ das pressões inicial e final estão apresentadas na tabela I.

Tabela I - Comparação das Pressões Inicial e Final Verificada nos Pacientes na Unidade de Terapia Intensiva e no Centro Cirúrgico, Mostrando que nos Dois Grupos a Pressão Inicial foi Significativamente maior que a Final nos Dois Grupos ( $p<0,001)$

\begin{tabular}{lcccc}
\hline & \multicolumn{2}{c}{$\begin{array}{c}\text { Terapia Intensiva }(\mathrm{n}=50) \\
\text { Grupo I }\end{array}$} & $\begin{array}{c}\text { Centro Cirúrgico }(\mathrm{n}=72) \\
\text { Grupo II }\end{array}$ \\
\hline Pressão em cmH & Inicial & Final & Inicial & Final \\
\hline $\mathrm{P}_{25}$ & 28,5 & 10 & 47 & 19 \\
Mediana & 42,5 & 15 & 69,5 & 25,5 \\
$\mathrm{P}_{75}$ & 65,5 & 19,5 & 108 & 32 \\
Média \pm DP & $56,2 \pm 48$ & $15,5 \pm 6,7$ & $85,3 \pm 56,3$ & $26,7 \pm 8,2$ \\
\hline
\end{tabular}

\section{DISCUSSÃO}

De acordo com vários autores, a pressão no interior do balonete é o fator mais importante na gênese da lesão traqueal pós-intubação traqueal. Estudos comprovam esta afirmação; entretanto, nenhum deles avaliou o grau de lesão em relação às pressões de insuflação do balonete ${ }^{1,2,9,10,17-21}$.

As médias das pressões iniciais de balonetes obtidas mostraram-se acima da pressão considerada ideal para boa perfusão na mucosa traqueal. Com a manobra preconizada foi possível diminuir acentuadamente a média de 85,3 para 26,7 (centro cirúrgico) e 56,2 para 15,5 (terapia intensiva). Com essas pressões médias, seguramente a pressão da mucosa deverá ser normal.

A diferença entre as médias das pressões iniciais dos grupos foi de $29,1 \mathrm{~cm} \mathrm{H}_{2} \mathrm{O}$. Isto deve ter ocorrido pelo fato dos profissionais preencherem os balonetes, geralmente, pela injeção de volume fixo de ar. Como os anestesiologistas costumam utilizar tubos com diâmetros internos mais próximos aos da traquéia, isto é, com o maior diâmetro interno possível, é fácil 
entender por que as pressões iniciais no grupo do centro cirúrgico tiveram média mais alta. Tendo em vista que a pressão final também mostrou diferença entre os grupos $(11,2 \mathrm{~cm}$ de água), outra possibilidade seria a utilização de balonetes com formatos diferentes nas duas Instituições, apesar dos tubos serem da mesma marca $\left(\right.$ Rush $\left.^{\circledR}\right)$. Se o balonete tiver formato mais alongado, terá maior superfície de contato com a mucosa traqueal, necessitando menor pressão para vedar o espaço entre a mucosa e o tubo de intubação traqueal. Este é um dos princípios seguido para o desenvolvimento dos balões de baixa pressão.

As comparações dos valores das pressões iniciais com as pressões finais após a manobra mostraram diferença estatisticamente significante. Isso comprova que a manobra realizada pode ser adequada para manter a pressão no interior do balão em nível baixo, permitindo ventilação adequada do paciente. Além disso, o desvio padrão das pressões finais dos dois grupos aproximou-se bastante, mostrando a capacidade da manobra em homogeneizar as pressões no interior dos balões.

Pacientes submetidos à anestesia geral utilizando-se o $\mathrm{N}_{2} \mathrm{O}$ merecem atenção especial. $\mathrm{O} \mathrm{N}_{2} \mathrm{O}$ difunde-se pela membrana do balonete distendendo-o e aumentando sua pressão. Deve-se monitorar a pressão do balonete durante a anestesia, até cessar o uso do $\mathrm{N}_{2} \mathrm{O}$, ou insuflá-lo com a mesma concentração de anestésico utilizada para ventilar o paciente 22-25. Em nosso estudo, excluiu-se os pacientes que estavam sendo anestesiados com $\mathrm{N}_{2} \mathrm{O}$, eliminando esta variável.

Magovern e col., em $1972^{2}$, com o auxílio da bioengenharia desenvolveram balonete com sistema externo regulador de pressão. Este sistema funciona de forma que mesmo insuflando-se volumes excessivos de ar no balonete há um balonete externo constituído de material extremamente elástico que se distende evitando elevações de pressão do balão acima de $30 \mathrm{cmH}_{2} \mathrm{O}$. Este tubo com sistema chamado de válvula reguladora de pressão é conhecido comercialmente como tubo de Lanz ${ }^{\circledR}$. Seria a solução do problema de controle da pressão do balonete ${ }^{26}$, mas é quase dez vezes mais caro do que o tubo convencional, ocorre ruptura do sistema com mais facilidade, necessitando de trocas mais freqüentes, e permite passagem de secreções orofaríngeas para a traquéia.

No Segundo Consenso Brasileiro de Ventilação Mecânica ${ }^{23}$, atribui-se à enfermagem os cuidados de verificação da pressão do balonete, no mínimo a cada 12 horas. Preconiza-se insuflação de volume necessário para evitar escape de ar e movimentação do tubo na traquéia com pressões inferiores a $25 \mathrm{mmHg}$. Concordamos em parte com essa orientação. Não concordamos com a movimentação, pois o balão não deve servir de fixação do tubo à traquéia.

Outros autores ${ }^{27}$ realizaram estudo para tentar estimar o volume de ar necessário no balonete, para cada tamanho do tubo, de forma que a pressão não fosse maior do que 20 a 30 $\mathrm{mmHg}$. Acreditamos que este volume depende da relação entre o diâmetro interno traqueal e externo do tubo. Como é difícil estimar com exatidão o diâmetro interno traqueal, a escolha do tubo é sempre subjetiva e depende do tamanho do espaço glótico. Por este motivo, a estimativa do volume de ar a ser insuflado no balonete não nos parece bom método. Alguns dos estudos experimentais e clínicos ${ }^{13,21,27,28}$ citam que a insuflação do balonete deve ser realizada até que não haja escape de ar, de acordo com nosso estudo. No entanto, nenhum deles descreve como realizar este procedimento. Estudo semelhante ao nosso recomenda controle da pressão do balonete, sendo necessária a disponibilidade de manômetro ${ }^{24}$. Acreditamos ter atingido nosso objetivo secundário, ou seja, descrever um método para enchimento do balonete diminuindo o trauma na traquéia, pois basta seguir os passos descritos no método para manter a pressão do balonete em nível baixo. Com uma seringa plástica convencional de $20 \mathrm{ml}$, seguindo os itens de a) a g) evitamos enchimento excessivo do balonete.

Alguns autores ${ }^{29}$ confirmaram a existência de boa relação do pico de pressão inspiratório com a pressão mínima necessária no balonete. Concluem que pacientes com pico de pressão de inspiração elevada têm maior possibilidade de apresentar estenose traqueal, por necessitar de maior pressão no balonete para evitar perda de parte do volume corrente.

Outros autores ${ }^{30}$ levantaram sérios problemas em relação aos balonetes de baixa pressão. Seu nível de pressão, para evitar grande agressão à mucosa traqueal (20 a $30 \mathrm{~cm}$ de água), pode permitir a passagem de fluido da orofaringe para a parte distal da traquéia, favorecendo complicações infecciosas traqueobrônquicas e pulmonares. Isto ocorre pela presença de pequenos canais que se formam entre a parede do balonete de baixa pressão (distensão parcial da circunferência do balonete) e a mucosa traqueal. Outro estudo ${ }^{31}$ realizado em modelo experimental com traquéias humanas de cadáveres, desenvolveu um balonete que evita a passagem de líquidos, com pressões similares às dos balonetes convencionais de baixa pressão com válvula reguladora de pressão. Este balonete é de látex, sendo distendido por completo quando cheio, sem permitir a formação de pregas em sua parede. A passagem de corante entre o balonete e a parede traqueal, que pode ocorrer no balonete convencional, não ocorre no balão de látex. Reali-Foster e col. ${ }^{32}$ testaram em carneiros dispositivo que substitui o balonete, obtendo bom controle da passagem de líquidos, com dano traqueal insignificante.

Yuong e col. ${ }^{33}$ testaram em traquéias de porco, balonete de tubo traqueal de material e formato especial que evita a passagem de líquidos, com pressão de insuflação baixa. Documentaram a passagem de líquidos no balonete convencional de baixa pressão nos animais. Colocando o tubo de intubação no interior de seringa de $20 \mathrm{~cm}^{3}$ com balonete convencional e com o balonete desenvolvido, observaram a mesma passagem de líquido. Colocaram estas na posição vertical com corante depositado na porção superior do balão. O corante passou entre a parede do balão convencional e da seringa. Em sua conclusão, relatam dúvidas quanto à necessidade de que o balonete desenvolvido necessite ter tamanho diferenciado, para tubos de maior ou menor diâmetro interno, como os de 9 e $7 \mathrm{~mm}$. 
Os tubos de intubação com balonetes acompanhadas de válvula reguladora de pressão (Lanz $\left.{ }^{\circledR}\right)$ ainda não têm seu uso padronizado na maioria dos hospitais, devido ao seu alto custo. Já o balão de Young e col. ${ }^{33}$ e o tubo com o dispositivo experimentado por Reali-Foster e col. ${ }^{32}$, até onde sabemos, não chegaram a ter uso clínico.

Já estão disponíveis no mercado tubos de intubação traqueal com sistema de aspiração contínua de secreção do líquido acumulado na porção superior do balão. Eles evitam o acúmulo de líquidos na porção proximal do balonete e sua aspiração pulmonar, com conseqüente diminuição da incidência de efeitos, como a broncopneumonia em pacientes intubados. Este mesmo tubo apresenta também válvula reguladora de pressão, o que diminui muito o trauma traqueal ${ }^{34}$.O problema continua sendo o preço destes tubos.

Conclui-se que os grupos de pacientes intubados na Terapia Intensiva e no Centro Cirúrgico apresentaram pressões no interior dos balonetes em níveis acima do necessário para a adequada ciclagem do respirador e sem a perda de volume de ar corrente. Amanobra padronizada neste estudo para diminuir a pressão no interior do balonete foi eficiente, simples, rápida e de baixo custo.

\section{Study of Tracheal Tube Intra-Cuff Pressure}

André Galante Alencar Aranha, M.D.; Vicente Forte, M.D.; João Aléssio Juliano Perfeito, M.D.; Luiz Eduardo Villaça Leão, M.D.; Carlos Jogi Imaeda, M.D.; Yara Juliano, M.D.

\section{INTRODUCTION}

Tracheal stenosis may have different origins, however the most frequent is related to tracheal intubation ${ }^{1-8}$.

The incidence of post intubation laryngeal and tracheal stenosis varies from $1.5 \%$ to $19 \%{ }^{9-11}$.

Cooper and Grillo have tested in dogs and posteriorly in men ${ }^{1}$ a tracheal tube with latex cuff, easily expandable and more flexible, that would adapt to tracheal shape needing less pressure to prevent air flowleakage. After the development of these low pressure cuff tubes, in the beginning of the 1970's, with the help of bioengineering, there has been a major decrease in post-intubation tracheal stenosis ${ }^{1,2,7,11}$. However, even low pressure cuffs, when excessively inflated, may be noxious to the trachea ${ }^{12,13}$.

Nordin, in 1977, has shown that tracheal tube cuff pressure above $30 \mathrm{mmHg}\left(40 \mathrm{cmH}_{2} \mathrm{O}\right)$ determines mucosal ischemia and has recommended that pressure should remain below 20 $\mathrm{mmHg}\left(26 \mathrm{cmH}_{2} \mathrm{O}\right)^{4}$.

Pippin et al., in 1983, have mailed a questionnaire to 152 intensive care units in the UK, asking whether pressure within tracheal tube cuffs was routinely measured. The answer was negative in $83.2 \%$ of questionnaires ${ }^{14}$.
Post-intubation tracheal stenosis may be present in patients under tracheal intubation with low pressure cuff tubes even for short periods up to few hours ${ }^{7,15,16}$.

Forte, in 1996, has published a study with 250 patients submitted to tracheoplasties in the period March 1969 to March 1996 for post-intubation tracheal stenosis correction and has attributed $80 \%$ of the lesions to tracheal tube cuffs ${ }^{7}$.

This study aimed at checking tracheal tubes intra-cuff pressures in intensive care unit and surgical patients, and at testing a simple, fast and cheap maneuver to maintain cuff pressure in minimum values and, whenever possible, below 30 $\mathrm{cmH}_{2} \mathrm{O}$.

\section{METHODS}

After the institutional approval of Hospital São Joaquim, Real e Benemérita Sociedade Portuguesa de Beneficência, 50 lntensive Care Unit patients (Group I) were randomly selected in the period July to December 1997. After the Hospital Professor Edmundo Vasconcelos Ethics Committee approval, a group of 72 surgical patients was selected in the period May to August 1999 (Group II).

Cuff pressures were checked in group I in random days. Tracheal tubes were of the Rush ${ }^{\circledR}$ brand, with 7 to $9 \mathrm{~mm}$ internal diameter, all with low pressure cuffs. In group II, 72 patients intubated for surgical procedures under general anesthesia were studied. Cuff pressures were also checked. Three out of seven operating rooms were randomly selected for the morning period and three for the afternoon period. Exclusion criteria were patients under local anesthesia or anesthetized with nitrous oxide, because it crosses cuff material changing pressure during anesthesia. All patients were intubated with Rush ${ }^{\circledR}$ low pressure cuff tubes.

Patients were intubated by different professionals in both groups. Air injection in the cuff at tracheal intubation and maintenance of filling level were performed according to personal criteria of professionals in charge who were blind to the study.

\section{Tracheal Tube Cuff Pressure Measurement Technique}

Pressures inside tracheal tube cuffs were measured with a manometer (P-Gauge, Mallinckrodt Medical ${ }^{\circledR}$ ). This device has a syringe graduated in $15 \mathrm{~cm}^{3}$, coupled to a digital manometer graduated in $\mathrm{cmH}_{2} \mathrm{O}$.

Intra-cuff pressure was measured as follows:

a) Patient's oropharyngeal secretion aspiration;

b) Connection of the device to external tube cuff;

c) Measurement of tracheal tube intra-cuff pressure, defined as initial pressure (P1);

d) Cuff was slowly deflated and, at the same time, the investigator maintained his external acoustic meatus 10 to 20 $\mathrm{cm}$ apart from patient's mouth. This way, the investigator could listen to the murmur sound when part of tidal volume leaked around the cuff in the inspiratory period of artificial ventilation; 
e) Then, cuff was slowly inflated until murmur was no longer heard;

f) Intra-cuff pressure needed to stop leakage was recorded and considered the final pressure (P2);

g) After air leakage interruption, air remaining in the syringe was measured and defined as unnecessary volume (V).

Time spent to complete all stages was recorded for the first 10 maneuvers.

Wilcoxon non-parametric test was used to compare initial and final intra-cuff pressures. In addition to median, $\mathrm{P}_{25}$ and $\mathrm{P}_{75}$, mean and standard deviation were also included to give an idea of data variation.

Null hypothesis rejection level was established in 0.05 or $5 \%$ $(p<0.05)$, being significant figures marked with a star $\left(^{*}\right)$.

\section{RESULTS}

Group I was composed of 18 females (36\%) and 32 males (64\%), aged 22 to 99 years (mean 62.1 years). Intubations were determined by cardiovascular surgeries (27 cases), bronchopneumonia (5 cases), postoperative period of general surgeries (5 cases), myocardial infarction (3 cases) and other causes (10 cases). Intubation time varied from a few hours to 23 days.

Group II was composed of 42 males (58.3\%) and 30 females $(41.7 \%)$, aged 15 to 68 years (mean 45.2 years). Intubation time varied from 100 to 380 minutes.

Time to perform all described stages to measure intra-cuff pressure was less than 3 minutes. Initial pressures were always higher than final pressures after the above-described maneuver. Figure 1 shows initial and final pressure values in Group I patients. Figure 2 shows initial and final pressures found in Group II patients. Figures show major initial pressure variations and, as a consequence, a high standard deviation. Only 12 Group I patients (24\%) had initial pressures lower than $30 \mathrm{cmH}_{2} \mathrm{O}$. All 50 patients $(100 \%)$ had air pressure and

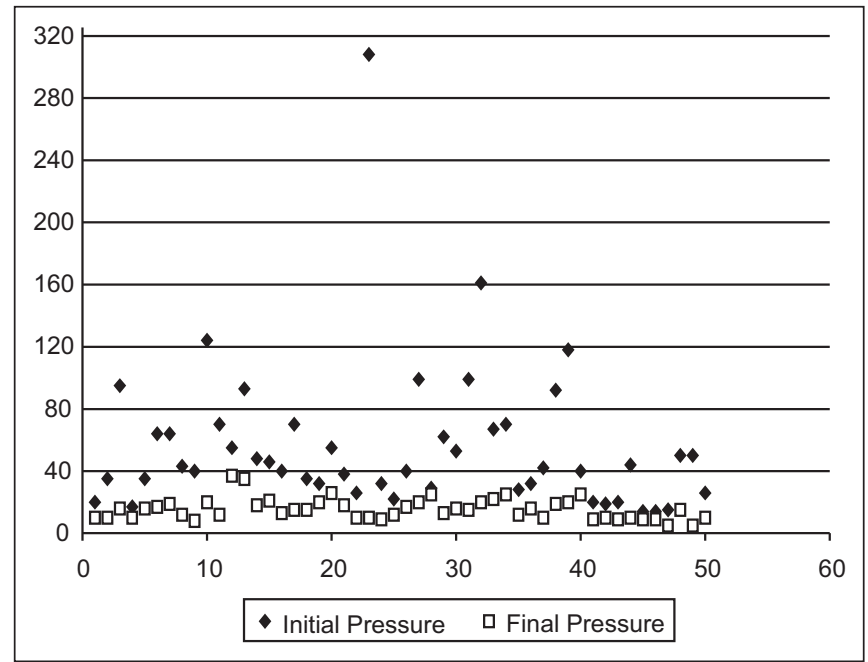

Figure 1 - Initial and Final Pressures, in $\mathrm{cmH}_{2} \mathrm{O}$, within Tracheal Tube Cuffs in Group I Patients

Revista Brasileira de Anestesiologia

Vol. 53, N 6, Novembro - Dezembro, 2003

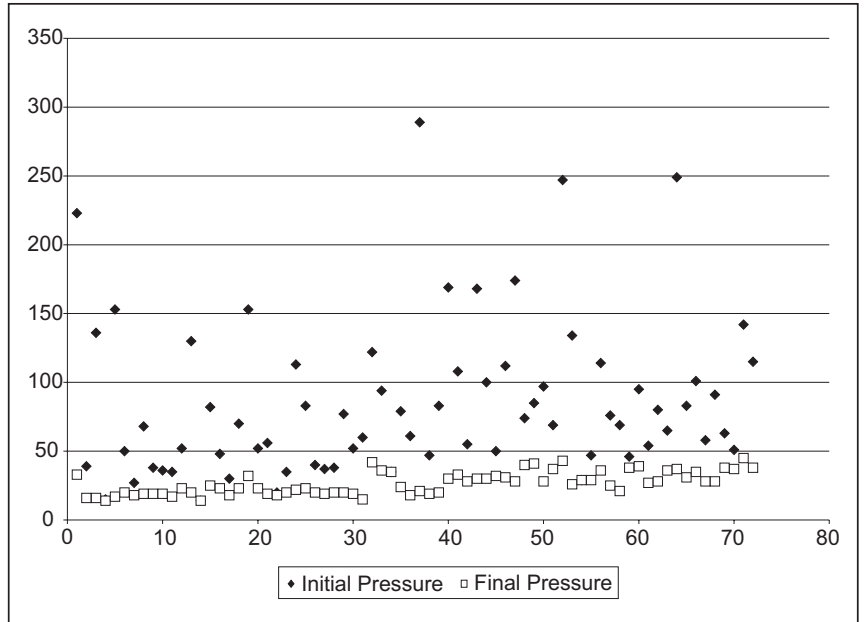

Figure 2 - Initial and Final Pressures, in $\mathrm{cmH}_{2} \mathrm{O}$, within Tracheal Tube Cuffs in Group II Patients

volume decrease inside the cuffs and only 2 patients have not reached pressures below $30 \mathrm{cmH}_{2} \mathrm{O}$ after the maneuver. In group II, five cuffs $\left(6.9 \%\right.$ ) were below $30 \mathrm{cmH}_{2} \mathrm{O}$. From 72 patients, there has been no cuff air removal in just $2(2.7 \%)$ and in 23 patients $(31.9 \%)$ pressures below $30 \mathrm{cmH}_{2} \mathrm{O}$ were not maintained without air leakage during ventilation.

Initial and final pressures mean, standard deviation, median, $\mathrm{P}_{25}$ and $\mathrm{P}_{75}$ are shown in table $\mathrm{I}$.

Table I - Comparison of Initial and Final Pressures in Intensive Care Unit and Operating Center Patients, Showing that in Both Groups, Initial Pressure was Significantly Higher than Final Pressure in Both Groups ( $p<0.001)$

\begin{tabular}{lcccc}
\hline & \multicolumn{2}{c}{$\begin{array}{c}\text { Intensive Care }(\mathrm{n}=50) \\
\text { Group I }\end{array}$} & \multicolumn{2}{c}{$\begin{array}{c}\text { Operating Center }(\mathrm{n}=72) \\
\text { Group II }\end{array}$} \\
\hline Pressure in $\mathrm{cmH}_{2} \mathrm{O}$ & Initial & Final & Initial & Final \\
\hline $\mathrm{P}_{25}$ & 28.5 & 10 & 47 & 19 \\
Median & 42.5 & 15 & 69.5 & 25.5 \\
$\mathrm{P}_{75}$ & 65.5 & 19.5 & 108 & 32 \\
Mean $\pm \mathrm{SD}$ & $56.2 \pm 48$ & $15.5 \pm 6.7$ & $85.3 \pm 56.3$ & $26.7 \pm 8.2$ \\
\hline
\end{tabular}

\section{DISCUSSION}

According to several authors, intra-cuff pressure is the most important factor for post-intubation tracheal injuries. Studies have confirmed such statement; however, no study has evaluated injuries as a function of cuff inflation pressures $1,2,9,10,17,21$

Mean initial cuff pressures obtained were always above the pressure considered optimal for a good tracheal mucosa perfusion. With the proposed maneuver, it was possible to significantly decrease mean from 85.3 to 26.7 (operating center) and from 56.2 to 15.5 (ICU). These mean pressures are safe to maintain normal mucosal pressure. 
The difference of mean initial pressures between groups was $29.1 \mathrm{~cm} \mathrm{H}_{2} \mathrm{O}$. This might have been due to the fact that in general, cuffs were filled by fixed air volume injection. Since anesthesiologists use tubes with internal diameters closer to tracheal diameter, that is, with the largest possible internal diameter, it is easy to understand why surgical patients initial pressures had a higher mean. Considering that final pressure was also different between groups ( $11.2 \mathrm{~cm}$ of water), another possibility would be the use of cuffs with different shapes in both institutions, although tubes were of the same brand $\left(\right.$ Rush $\left.^{\circledR}\right)$. If the cuff is longer, there is more contact surface with tracheal mucosa, needing less pressure to seal the space between the mucosa and the tracheal tube. This is one of the principles adopted for the development of low-pressure cuffs.

Initial and final pressure comparisons have shown statistically significant differences. This proves that the maneuver may be adequate to maintain low intra-cuff pressure, allowing adequate patient's ventilation. In addition, final pressure standard deviation was similar for both groups, showing the ability of the maneuver to homogenize intra-cuff pressures.

Patients submitted to general anesthesia with $\mathrm{N}_{2} \mathrm{O}$ deserve special attention. $\mathrm{N}_{2} \mathrm{O}$ spreads through cuff's membrane expanding it and increasing its pressure. Cuff pressure should be monitored during anesthesia until $\mathrm{N}_{2} \mathrm{O}$ withdrawal, or cuff should be inflated with the same anesthetic concentration used to ventilate patients ${ }^{22-25}$. In our study, patients receiving $\mathrm{N}_{2} \mathrm{O}$ were excluded, thus eliminating such variable.

Magovern et al., in $1972^{2}$, have developed with the help of bioengineering a cuff with external pressure regulator. This system works in a way that even when excessive volumes are inflated, there is an external cuff made of extremely elastic material which expands preventing cuff pressure increases above $30 \mathrm{cmH}_{2} \mathrm{O}$. This tube with a system called pressure-regulating valve, is commercially known as Lanz ${ }^{\circledR}$ tube. This would be the solution to control intra-cuff pressures ${ }^{26}$, but it is almost 10 -fold more expensive than conventional tubes, there is more system rupture needing more frequent replacements, and it allows the passage of oropharyngeal secretions to the trachea.

The Second Brazilian Consensus of Mechanical Ventilation ${ }^{23}$ assigns to the nursing team the responsibility to check intra-cuff pressure at least at every 12 hours. It is recommended a volume inflation needed to prevent air leakage and tube movement in the trachea with pressure below 25 $\mathrm{cmH}_{2} \mathrm{O}$. We partially agree with this orientation. We do not agree with the movement, because the cuff should not fix the tube to the trachea.

Other authors ${ }^{27}$ have carried out a study to estimate cuff air volume needed for different tube sizes, in a way that pressure would never be higher than 20 to $30 \mathrm{mmHg}$. We believe that this volume depends on the ratio between internal tracheal diameter and external tube diameter. Since it is difficult to accurately estimate internal tracheal diameter, tube choice is always subjective and depends on the glottic space size. So the estimate of air volume to be inflated in the cuff is not a good method, in our opinion.
Some experimental and clinical trials ${ }^{13,21,27,28}$ mention that cuffs should be inflated until there is no longer air leakage, in line with our study. However, none of them describes how to perform the procedure. A study similar to ours recommends cuff pressure control and requires a manometer ${ }^{24}$. We believe that we have reached our secondary objective, which was to describe a method to fill the cuff decreasing tracheal trauma, because it is enough to follow the described steps to maintain low intra-cuff pressures. With a conventional $20 \mathrm{ml}$ syringe, and following steps a) to g), we have prevented excessive cuff filling.

Some authors ${ }^{29}$ have confirmed a good ratio between inspiratory pressure peak and minimum pressure needed in the cuff. They have concluded that patients with high inspiration peak pressures have a higher possibility of presenting tracheal stenosis because they need higher cuff pressures to prevent partial tidal volume leakeage.

Other authors ${ }^{30}$ have raised major problems with low-pressure cuffs. Their pressure level to prevent major tracheal mucosa aggression (20 to $30 \mathrm{~cm}$ of water) may allow oropharyngeal fluids to reach the distal trachea, favoring infectious tracheobronchial and pulmonary complications. This is due to the presence of small channels between low-pressure cuff walls (partial cuff circumference distention) and the tracheal mucosa. A different study ${ }^{31}$ in an experimental model with human tracheas of cadavers has developed a cuff which prevents fluid leakage with pressures similar to conventional low pressure cuffs with pressure regulating valve. This cuff is made of latex and is totally distended when inflated preventing the presence of folds on its wall. Dye leakage between the cuff and the tracheal wall, which may occur with conventional cuffs, is not present in the latex cuff. Reali-Foster et al. ${ }^{32}$ have tested in ewes a device to replace cuffs, with good control on fluid leakage and minor tracheal damage.

Young et al. ${ }^{33}$ have tested in swine tracheas a cuff of special material and shape which prevents fluid leakage with low inflation pressures. They have documented fluid leakage in conventional and low pressure cuffs in animals. Placing the tube inside a $20 \mathrm{~cm}^{3}$ syringe with conventional cuffs and their new cuff, they have observed the same fluid leakage. These were placed in the vertical position with the dye placed in the upper part of the cuff. Dye has leaked between conventional cuff wall and the syringe. In their conclusion, they question the need for the new cuff to have differentiated size, and for higher or lower internal diameter tubes, such as 9 and $7 \mathrm{~mm}$. Tubes with cuffs and pressure regulating valve $\left(\right.$ Lanz $\left.^{\circledR}\right)$ are still not standardized in most hospitals due to their high cost. Yuong et al. ${ }^{33}$ tube, and the tube with the device tested by Reali-Foster et al. ${ }^{32}$, to our knowledge, have never been clinically used.

There are in the market tracheal tubes with a system for continuous aspiration of fluids accumulated in the upper part of the cuff. They prevent fluid build-up in cuff's proximal part and its pulmonary aspiration, with consequent decrease in the incidence of effects, such as bronchopneumonia, in intubated patients. This same tube also has pressure-regulating valve, 
which decreases tracheal trauma ${ }^{34}$. The problem is still the price of such tubes.

We concluded that patients intubated in Intensive Care Units and Operating Centers had intra-cuff pressures higher than needed for adequate ventilator cycling without air volume loss. The maneuver standardized in this study to decrease intra-cuff pressure was effective, simple, fast and cheap.

\section{REFERÊNCIAS - REFERENCES}

01. Grillo HC, Cooper JD, Geffin et al - A low-pressure cuff for tracheostomy tubes to minimize tracheal injury. J Thorac Cardiovasc Surg, 1971;62:898-907.

02. Mogovern GJ, Shively JG, Fecht D et al - The clinical and experimental evolution of a controlled-pressure intratracheal cuff. J Thorac Cardiovasc Surg, 1972;64:747-756.

03. Nordin $U$ - The trachea and cuff-induced tracheal injury. Acta Otolaryngol, 1977;345:1-71.

04. Nordin $U$ - The regeneration after cuff-induced tracheal injury. Acta Otolaryngol, 1982;94:541-555.

05. Ferdinende P, Kim D - Prevention of posintubation laryngotracheal stenosis. Acta Otorhinolarymgol Belg, 1995;49: 341-346.

06. Grillo HC, Donahue DM, Mathisen DJ et al - Posintubation tracheal stenosis. J Thorac Cardiovasc Surg, 1995;109:486-493.

07. Forte $V$ - Ressecção da estenose traqueal pós-intubação com reconstrução da traquéia por anastomose laringo, crico ou traqueotraqueal: análise clínica e cirúrgica. (Tese - Docência Universidade Federal de São Paulo Escola Paulista de Medicina) São Paulo, 1996.

08. Goldberg M, Pearson FG - Pathogenesis of tracheal stenosis following tracheostomy with a cuff tube. Thorax, 1972;27: 670-692.

09. Stauffer JL, Olson DE, Petty TL - Complications and consequences of endotracheal intubation and tracheotomy. Am J Med, 1981;70:65-76.

10. Kastanos N, Miró RE, Perez AM et al - Laryngotracheal injury due to endotracheal intubation: Incidence, evolution, and predisposing factors. A prospective long-term study. Crit Care Med, 1983;11:362-367.

11. Pearson FG - Thoracic Surgery. New York, Churchill Livingstone, 1995;251.

12. Lewis FR, Schlobohm RM, Thomas AN - Prevention of complications from prolonged tracheal intubation. Am J Med, 1978; 135:452-457.

13. Oliveira C, Ferreira CAS, Feltrim MIZ et al - Avaliação da pressão do tubo endotraqueal nas primeiras horas do pós-operatório imediato de cirurgia cardíaca. Rev Bras Terap Intensiva, 1992;4:116-118.

14. Pippin LK, Bawes JB - Long-term tracheal intubation practice in the United Kingdom. Anaesthesia, 1983;38:791-795.

15. Messahel BF - Total tracheal obliteration after intubation with a low- pressure cuffed traqueal tube. $\mathrm{Br} \mathrm{J}$ Anaesth, 1994;73: 697-699.

16. Yang KL - Tracheal stenosis after a brief intubation. Anesth Analg, 1995;80:625-627.

17. Cooper JD, Grillo HC - The evolution of tracheal injury due to ventilatory assistance through cuffed tubes: a pathologic study. Ann Surg, 1969;169:334-348.

18. Bryce DP, Briant TDR, Pearson FG - Laryngeal and tracheal complications of intubations. Ann Otol, 1968;77:442-461.

Revista Brasileira de Anestesiologia

Vol. 53, № 6, Novembro - Dezembro, 2003
19. Cooper JD, Grillo HC - Experimental production and prevention of injury due to cuffed tracheal tubes. Surg Gynecol Obstet, 1969;129:1235-1241.

20. Donnelly WH - Histopathology of endotracheal intubation. Arch Path, 1969;88:511-520.

21. Martins RHG, Braz JRC, Bretan? et al - Lesões precoces da intubacão endotraqueal. Rev Bras Otorrinolaringol, 1995;61: 1-4.

22. Stanley TH, Liu W - Tracheostomy and endotracheal tube cuff volume and pressure changes during thoracic operations. Ann Thorac Surg, 1975;20:145-151.

23. Passos E, Castilho VG - Papel da enfermagem na assistência ao paciente em ventilação mecânica. J Pneumol, 2000;26: 27-34.

24. Tu HN, Saidi N, Lieutaud T et al - Nitrous oxide increases endotracheal cuff pressure and the incidence of tracheal lesions in anesthetized patients. Anesth Analg, 1999;89:187-190.

25. Braz JRC, Navarro LHC, Takata IH et al - Endotracheal tube cuff pressure: need for precise measurement. Med J Rev Paul Med, 1999;117:243-247.

26. Navarro LHC, Braz JRC, Pletsch AK et al - Estudo camparativo das pressões dos balonetes de tubos traqueais contendo ou não valvula reguladora de pressão de Lanz. Rev Bras Anestesiol, 2001;51:17-27.

27. Barbosa PMK, Santos BMO - Determinação do volume de ar no "cuff" de sondas endotraqueais. R Bras Enferm, 1996;49: 225-238.

28. Bernhard WN, Yost L, Joynes $D$ et al - Intracuff pressure in endotracheal and tracheostomy tubes. Chest, 1985;87: 720-725.

29. Guyton DC, Barlow MR, Besselievre TR - Influence of airway pressure on minimum occlusive endotracheal tube cuff pressure. Crit Care Med, 1997;25:91-94.

30. Young PJ, Rollinson M, Downward G et al - Leakage of fluid past the tracheal tube cuff in a benchtop model. $\mathrm{Br} \mathrm{J}$ Anaesth, 1997;78:557-562.

31. Young PJ, Ridley AS, Downward G - Evaluation of design tracheal tube cuff to prevent leakage of fluid to the lungs. $\mathrm{Br} \mathrm{J}$ Anaesth, 1998;80:796-799.

32. Reali-Forster C, Kolobow T, Giacomini M et al - New ultrathin-walled endotracheal tube with a novel laryngeal seal design. Anesthesiology, 1996;84:162-172.

33. Young PJ, Blunt MC - Improving the shape and compliance characteristics of a high-volume, low-pressure cuff improves tracheal seal. Br J Anaesth, 1999;83:887-889.

34. Valles J, Artigas A, Rello $\mathrm{J}$ et al - Continuos aspiration of subglotic secretions in preventing ventilator associated pneumonia. Ann Intern Med, 1995;122:179-186.

\section{RESUMEN}

Aranha AGA, Forte V, Perfeito JAJ, Leão LEV, Imaeda CJ, Juliano Y -Estudio de las Presiones en el Interior de los Balones de Tubos Traquéales

JUSTIFICATIVA Y OBJETIVOS: Como no es de rutina el control de la presión en el interior de los balones de tubos traquéales, y como también no hay descripción detallada en la literatura de como mantenerla abajo de los $30 \mathrm{cmH}_{2} \mathrm{O}$ sin utilización de manómetro, se decidió confirmar las presiones en el interior de los balones de tubos traquéales en pacientes bajo intubación traqueal en la unidad de terapia intensiva y en el centro quirúrgico, ensayando maniobras para mantener la presión en el balón abajo de $30 \mathrm{cmH}_{2} \mathrm{O}$, más en niveles mínimos 
necesarios para el ciclo del ventilador sin pérdida del volumen corriente.

MÉTODO: Se estudiaron las presiones en el interior de balones de tubos traquéales de 50 pacientes bajo intubación traqueal en la unidad de terapia intensiva (Grupo I) y 72 pacientes bajo intubación traqueal en el centro quirúrgico (Grupo II). Se experimentó una maniobra para obtener la presión mínima en el interior del balón del tubo traqueal, necesaria para una adecuada ventilación, sin vaciamiento de aire. Se registró la presión inicial $(P 1)$ en el interior de los balones de los tubos traquéales utilizándose un manómetro digital graduado en centímetros de agua, acoplado a una jeringa de $15 \mathrm{ml}$. Fue aspirada secreción de la orofaringe. Con el meato acústico externo del examinador próximo de la boca del paciente entre 10 y $20 \mathrm{~cm}$, se conectó el manómetro al balón, que fue vaciado lentamente, hasta escucharse ruido en soplo, por el vaciamiento del volumen corriente en el período inspiratorio de la ventilación artificial. En este momento, se llenó lentamente el balón hasta el desaparecimiento del ruido. Se anotó la presión final (P2) del balón y el volumen de aire que quedó en la jeringa del manómetro $(V)$.

RESULTADOS: Las medias de las presiones $P 1$ en los grupos I y II fueron 85, 3 y $56,2 \mathrm{cmH}_{2} \mathrm{O}$, respectivamente. Las medias de presiones $P 2$ en los grupos I y II fueron 26,7 y 15,5 $\mathrm{cmH}_{2} \mathrm{O}$ respectivamente. Después de la maniobra hecha, el desvío patrón bajó de 56,3 para 8,2 en el grupo l, y de 48 para 6,7 en el grupo II. En el grupo I, la maniobra redujo el volumen y la presión del balón en $100 \%$ de los pacientes y en el grupo II, en $97,3 \%$.

CONCLUSIONES: Los dos grupos presentaron presiones en el interior de los balones en niveles arriba de lo necesario para el ciclo del ventilador sin pérdida del volumen corriente. La maniobra para mantener la presión en el interior del balón en niveles inferiores a $30 \mathrm{cmH}_{2} \mathrm{O}$ fue simples y de pequeño costo. 